\title{
Líquen plano oral associado a fatores psicogênicos: Relato de caso
}

\author{
Oral Plan associated with psychogenic factors: Case report \\ Linquen Plano oral asociado a factorespsicógenos: Reporte de caso
}

Recebido: 21/02/2021 | Revisado: 01/03/2021 | Aceito: 15/03/2021 | Publicado: 21/03/2021

\author{
Isabella Fernanda de Melo Vasco \\ ORCID: https://orcid.org/0000-0002-8367-7257 \\ Universidade Federal de Alagoas, Brasil \\ E-mail: bellinhananda@hotmail.com \\ Sybelle Souza Oliveira Malta \\ ORCID: https://orcid.org/0000-0001-7917-2208 \\ Universidade Federal de Alagoas, Brasil \\ E-mail: sybellemalta@gmail.com \\ Carlos Vinícius Ramos Leão de Oliveira \\ ORCID: https://orcid.org/0000-0001-7134-1011 \\ Universidade Federal de Alagoas, Brasil \\ E-mail: carlosviniciustlc@hotmail.com \\ Kelly Rodrigues Mota \\ ORCID: https://orcid.org/0000-0002-1656-608X \\ Universidade Federal de Alagoas, Brasil \\ E-mail: kellyrmota@outlook.com \\ Islane Caroline Ferreira da Silva \\ ORCID: https://orcid.org/0000-0001-7705-0493 \\ Universidade Federal de Alagoas, Brasil \\ E-mail:Karolineferreiraodonto@gmail.com \\ Luiz Carlos Oliveira dos Santos \\ ORCID: https://orcid.org/0000-0002-7021-5491 \\ Universidade Federal de Alagoas, Brasil \\ E-mail: 1cos1@hotmail.com
}

\begin{abstract}
Resumo
O líquén plano oral é uma doença mucocutânea, de curso crônico, responsável por destruir a camada basal do epitélio e, quando não controlada, predispões à malignização. Apesar da sua etiologia incerta, o estresse e a ansiedade têm sido frequentemente evidenciados como fatores promotores para essa patologia. Dessa forma, esse estudo tem por objetivo relatar um caso de um paciente masculino com Líquen plano oral (LPO) associado a fatores psicogênicos. Paciente do gênero masculino, leucoderma, 32 anos, procurou atendimento odontológico com a queixa principal de que apresentava uma mancha branca na língua, que ardia ao se alimentar, e duração de um ano. Durante a anamnese, o paciente relatou não possuir hábitos nocivos e nem fazer uso de medicações controladas, todavia apresentava histórico de estresse e ansiedade devido à recente alteração na dinâmica de sua vida pessoal. Ao exame físico intraoral foram observadas lesões brancas na região lateral da língua e região de trígono retromolar direitas. As hipóteses clínicas de leucoplasia e líquen plano foram sugeridas e uma biópsia incisional foi realizada, sendo a peça cirúrgica encaminhada para análise histopatológica. Os achados microscópicos evidenciaram hiperceratose, acantose, corpos de Civatte e um infiltrado inflamatório crônico contendo linfócitos, macrófagos e plasmócitos, levando ao diagnóstico final de LPO. Ademais, o paciente foi orientado a buscar apoio psicológico profissional. O paciente foi acompanhado por um período de 2 meses e, durante o seu retorno, foi observada recuperação tecidual, sem sintomatologia ou recidiva. Portanto, fatores psicogênicos, como estresse e ansiedade, podem exercer influência negativa referente à exacerbação e surgimento do líquen plano oral.
\end{abstract}

Palavras-chave: Líquen plano bucal; Leucoplasia oral; Angústia psicológica; Manifestações bucais.

\begin{abstract}
Oral lichen planus is a chronic mucocutaneous disease responsible for destroying the basal layer of the epithelium and, when not controlled, predisposing to malignancy. Despite its uncertain etiology, stress and anxiety have often been highlighted as promoting factors for this pathology. Thus, this study aims to report a case of a male patient with oral lichen planus (OLP) associated with psychogenic factors. Male patient, leucoderma, 32 years old, sought dental care with the main complaint that he had a white spot on his tongue, which burned when eating, and lasted for one year. During the anamnesis, the patient reported not having harmful habits or using controlled medications, however, he had a history of stress and anxiety due to the recent change in the dynamics of his personal life. On intraoral physical examination, white lesions were observed in the lateral region of the tongue and in the region of the right retromolar triangle. The clinical hypotheses of leukoplakia and lichen planus were suggested and an incisional biopsy
\end{abstract}


was performed, and the surgical specimen was sent for histopathological analysis. Microscopic findings showed hyperkeratosis, acanthosis, Civatte bodies and a chronic inflammatory infiltrate containing lymphocytes, macrophages and plasma cells, leading to the final diagnosis of OLP. In addition, the patient was instructed to seek professional psychological support. The patient was followed up for a period of 2 months and, during his return, tissue recovery was observed, without symptoms or recurrence. Therefore, psychogenic factors, such as stress and anxiety, can have a negative influence on the exacerbation and appearance of oral lichen planus.

Keywords: Oral lichen planus; Oral leukoplakia; Psychological distress; Oral manifestations.

\section{Resumen}

El liquen plano oral es una enfermedad mucocutánea crónica responsable de destruir la capa basal del epitelio y, cuando no se controla, predispone a la malignidad. A pesar de su etiología incierta, el estrés y la ansiedad a menudo se han destacado como factores promotores de esta patología. Así, este estudio tiene como objetivo reportar un caso de un paciente masculino con liquen plano oral (LPO) asociado a factores psicógenos. Paciente de sexo masculino, leucoderma, de 32 años de edad, acudió al dentista con el principal síntoma de que presentaba una mancha blanca en la lengua, que le quemaba al comer y se prolongaba por un año. Durante la anamnesis, el paciente refirió no tener hábitos nocivos ni utilizar medicamentos controlados, sin embargo, tenía antecedentes de estrés y ansiedad por el cambio reciente en la dinámica de su vida personal. En el examen físico intraoral se observaron lesiones blancas en la región lateral de la lengua y en la región del triángulo retromolar derecho. Se sugirieron las hipótesis clínicas de leucoplasia y liquen plano, se realizó biopsia incisional y se envió la pieza quirúrgica para análisis histopatológico. Los hallazgos microscópicos mostraron hiperqueratosis, acantosis, cuerpos de Civatte y un infiltrado inflamatorio crónico conteniendo linfocitos, macrófagos y células plasmáticas, lo que llevó al diagnóstico definitivo de LPO. Además, se le indicó al paciente que buscara apoyo psicológico profesional. El paciente fue seguido por un período de 2 meses y, durante su regreso, se observó recuperación de tejido, sin síntomas ni recurrencia. Por tanto, factores psicógenos, como el estrés y la ansiedad, pueden influir negativamente en la exacerbación y aparición del liquen plano oral.

Palabras clave: Liquen plano oral; Leucoplasia oral; Estrés emocional; Manifestaciones estomatológicas.

\section{Introdução}

O líquen plano oral (LPO) é uma doença mucocutânea, de curso crônico, responsável por destruir a camada basal do epitélio e, quando não controlada, predispõe à malignização (Volkweis et al., 2015; Vilanova et al., 2012). Podendo se expressar desde as formas papular, em placa, bolhosa e reticular às formas mais preocupantes, como a atrófica ou erosiva, necessita de atenção especial, uma vez que apresenta características específicas (Werneck et al., 2016; Shirauna, 2014).

Com predisposição a se manifestar em mucosa jugal, língua e gengiva, o LPO pode vir acompanhado pela sensação de rugosidade, prurido e glossopirose (Robledo-Sierra et al., 2018) e, para isso, a corticoterapia é considerada o tratamento de escolha, podendo variar de administração, tópico ou sistêmico, a depender da gravidade da lesão (Schenkel et al., 2017). Nesse sentido, torna-se imprescindível o diagnóstico diferencial do LPO com outras lesões semelhantes da cavidade oral, como a reação liquenóide (RLO), a fim de determinar um tratamento específico e adequado (Riga-Neto et al., 2013).

Infelizmente, a sua etiologia ainda é incerta, entretanto, evidências mostram que se trata de uma doença autoimune relacionada a apoptose das células basais do epitélio oral e mediada pelas células T (Sugerman et al., 2002). Associado a isso, o estresse e a ansiedade têm sido frequentemente evidenciados como fatores promotores para essa patologia. (Kurago, 2016; Boorghani et al., 2010).

Dessa forma, a partir do momento em que há um desequilíbrio emocional, alterações na homeostase sistêmica ocorrem, afetando diretamente a imunidade por meio da estimulação de processos inflamatórios (Fitzpatrick et al., 2014; Tomaz et al., 2015). Assim, o uso de medicações, como benzodiazepínicos ou tricíclicos, pode ser recomendado para o tratamento de doenças psicogênicas, a fim de controlar a frequência de manifestações e recidivas do LPO (Mezzari et al., 2015; Pokupec, et al., 2009; Aguiar et al., 2011; Medwar Matheus, 2012).Visto posto, esse estudo tem por objetivo relatar um caso clínico de LPO reticular associado a fatores psicogênicos, com enfoque no seu diagnóstico e tratamento. 


\section{Metodologia}

Este estudo possui caráter observacional, qualitativo, descritivo, do tipo relato de caso (Pereira et al., 2018), no qual foi investigado um caso clínico de LPO reticular associado aos fatores de psicogênicos de estresse e ansiedade.

Esse estudo foi aprovado pelo Comitê de Ética em Pesquisa da Universidade Federal de Alagoas - UFAL (Parecer 4.297.866.), obedecendo a Resolução n. ${ }^{\circ}$ 466/2012 da Comissão Nacional de Ética em Pesquisa e em consonância com a Declaração de Helsinki.

Um Termo de Consentimento Livre e Esclarecido (TCLE) abordando os aspectos éticos, esclarecimentos sobre riscos, benefícios e prognósticos foi fornecido ao paciente e o mesmo autorizou a realização do procedimento mediante assinatura de tal documento.

\section{Relato de Caso}

Paciente do gênero masculino, leucoderma, 32 anos, compareceu a Clínica de Estomatologia da Universidade Federal de Alagoas - FOUFAL, com a queixa principal de que apresentava uma mancha branca na língua, que ardia ao se alimentar, e duração de um ano.

Durante a anamnese, o paciente relatou se consultar com médicos e dentistas anteriores e apenas a hipótese diagnóstica fúngica foi suspeita, a partir disso, antifúngicos foram prescritos e melhora na higiene oral foi orientada, entretanto, não houve sucesso na resolução do caso. Ademais, o paciente não apresentava hábitos nocivos, não era usuário de medicamentos e não apresentava restaurações dentárias em contato com as regiões de acometimento, motivos que, por ventura, explicassem o aparecimento dessas reações liquenóides, todavia apresentava histórico de estresse e ansiedade devido à recente alteração na dinâmica de sua vida pessoal.

Dessa forma, o Inventário de Ansiedade Traço-Estado (Biaggio et al., 1979) e a Escala de Rastreamento populacional para depressão (Silveira et al., 1998) foram aplicados a fim de obter o score de ansiedade e categorizá-lo. Após avaliação, o paciente pontuou 59 pontos, sendo categorizado como ansiedade de grau elevada.

Ao exame físico intraoral foram observadas lesões brancas na região lateral direta da língua (Figura 1A) e região de trígono retromolar direita (1B). Associado a isso, ao exame extraoral, os linfonodos submandibulares bilaterais apresentavamse palpáveis, móveis, de consistência lisa e fibroelástica, com ausência de dor.

Figura 1: (A) Lesão em borda lateral direita da língua; (B) Lesão reticular em região de trígono retromolar direita.
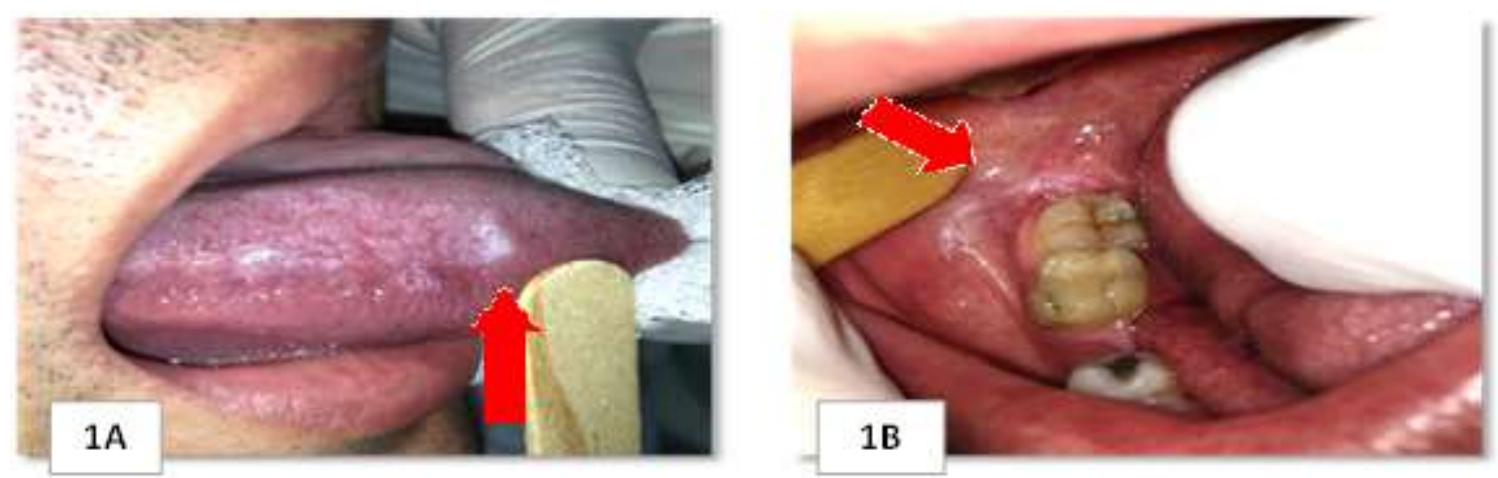

Fonte: Autores. 
Na Figura 1A, é interessante observar a presença de uma placa branca, plana, de contornos irregulares, superfície rugosa, em formato de rendilhado e medindo $3,5 \mathrm{~cm}$, na borda lateral direita da língua, que vinha acompanhada de sintomatologia dolorosa. Já na Figura 1B, a região do trígono retromolar direita foi acometida por uma lesão também branca, plana, de contornos irregulares e formato rendilhado, entretanto, apresentava superfície lisa e não acompanhava sintomatologia dolorosa. As hipóteses clínicas de leucoplasia e líquen plano foram sugeridas, e a realização de uma biópsia incisional foi realizada (Figura 2). A peça cirúrgica foi enviada para exame histopatológico.

Figura 2: Biópsia incisional da lesão na borda lateral direita da língua.

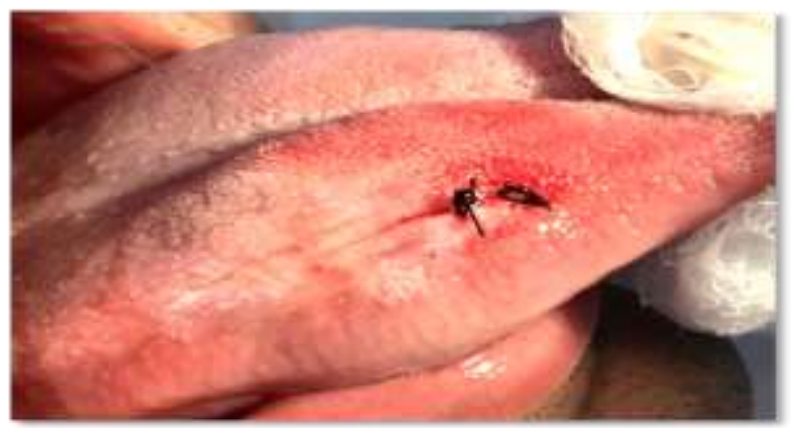

Fonte: Autores.

Nesse caso apresentado, a análise histopatológica revelou hiperceratose, acantose, corpos de Civatte, como também a lâmina própria subjacente exibindo infiltrado inflamatório crônico contendo linfócitos, macrófagos e plasmócitos. Dessa forma, esses achados foram condizentes com o diagnóstico final de Líquen Plano Oral.

Após procedimento cirúrgico, o paciente foi encaminhado a um psicólogo, a fim de controlar os fatores psicogênicos de estresse e ansiedade, e orientado a evitar o consumo de alimentos ácidos e condimentados, principalmente em fases sintomáticas. O mesmo foi acompanhado por um período de 2 meses e, durante o seu retorno, foi observada recuperação tecidual no local da lesão lingual, sem sintomatologia e recidivas (Figura 3A). Além disso, no mesmo período, houve também cicatrização da lesão em região de Trígono retromolar direita (Figura 3B).

Figura 3: (A) Pós-operatório de 2 meses; (B) Regressão da lesão reticular em região de trígono retromolar direita.

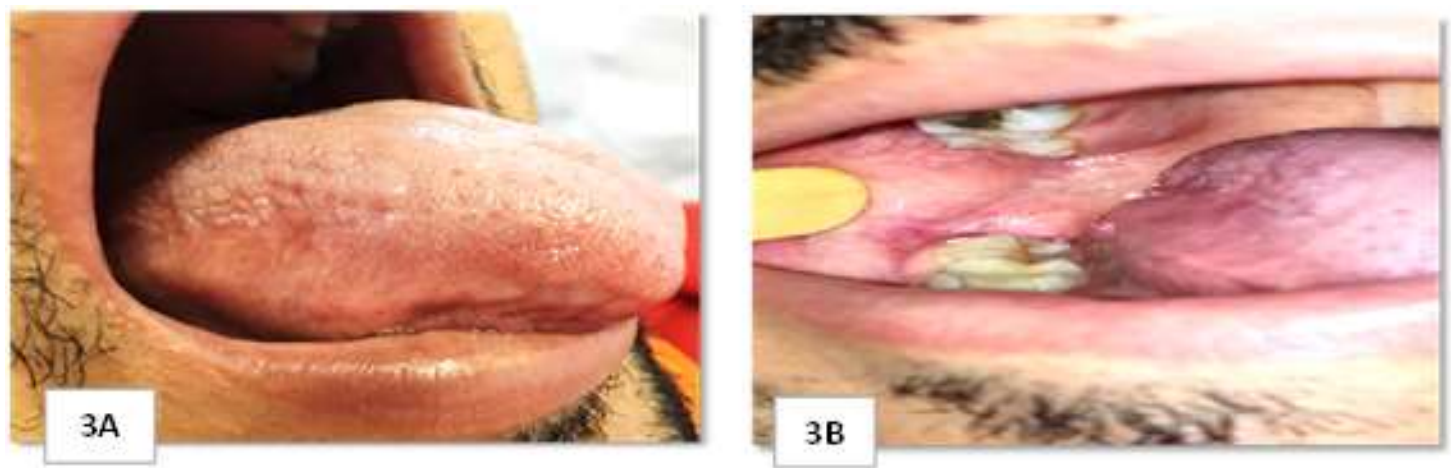

Fonte: Autores.

\section{Discussão}

As lesões bucais associadas à psicogênese baseadas na realidade integral do homem, que relaciona o físico ao psíquico, evidencia uma relação de exacerbação nas lesões bucais ao estresse, dando ênfase ao líquen plano como uma doença 
de particular interesse para o cirurgião dentista, uma vez o estresse psicológico e as modificações psiquiátricas podem alterar as funções imunológicas. Os métodos curativos atualmente utilizados para o tratamento do líquen plano da mucosa bucal apresentam resultados pouco satisfatórios, sendo fundamentalmente relevante para o êxito do tratamento, a consolidação emocional do paciente (Hampf et al., 1987).

Os sinais clínicos relacionados à sintomatologia podem ser resultantes ou intensificados por condições emocionais do paciente, como a ansiedade e a depressão, visto que fatores estressores podem ser responsáveis por respostas autoimunes, os quais têm sido considerados como fatores contribuintes para o agravamento do LPO (Chaudhary, 2004). Estudos apontam que transtorno de ansiedade e depressão se mostram mais acentuados em pacientes com LPO (Vallejo, Huerta et al., 2001).

Estudos sugerem que existe uma veemente correspondência entre os indivíduos portadores de LPO com um elevado grau de depressão, ansiedade e estresse, o que se porta de uma essencial relevância a recognição de tal estado emocional na prática clínica do profissional para um apropriado aconselhamento de tratamento e acompanhamento dos pacientes (Lundqvist et al., 2006).

Fatores como problemas financeiros, trabalhistas, familiares e morte em membros da família podem desencadear transtornos emocionais, os quais também são observados em pacientes portadores de LPO. Concluindo que estresse está estreitamente alusivo ao LPO, sugerindo dessa forma um fulcro psicológico aos pacientes associadas a tratamentos convencionais para líquen plano oral (Hirota, 2007). A convergência de fatores estressores, que permitiram validar o paciente como portador de ansiedade, foram primariamente uma anamnese arguitiva a qual além de portar informações de praxe, continha também uma arguição voltada à busca de fatores relacionados a níveis de estresse, ansiedade e depressão portando também de um exame clínico visual minucioso. Conectando os resultados obtidos com tais procedimentos foi possível concluir que o paciente portava um grau elevado no estado de ansiedade, sugerindo assim, uma correlação dos fatores psicogênicos com uma maior intensificação nas manifestações das lesões de LPO, sendo tais decorrentes da ação autoimune do sistema de defesa do indivíduo, visto que os cursos das respostas autoimunes podem ser acionados ou acelerados por fatores estressores, exacerbando dessa forma as lesões de LPO já preexistentes no indivíduo. Ademais, o paciente ainda salientou mudanças na rotina de vida, emigrando de uma cidade para outra com a finalidade de prestar cuidados a um familiar doente, podendo ser este o fator acionador de tais repercussões clínicas.

O aspecto histopatológico característico do LPO é a vacuolização da camada basal acompanhada por infiltrado linfocitário disposto em faixa superficial, junto a alterações das camadas córnea como hiperceratose, ortoceratose ou paraceratose, na camada granulosa ocorre hipergranulose por vezes em cunha, ceratinócitosapoptóticos chamados de corpos de Civatte e alterações da membrana basal como fragmentação e espessamento (Eisenberg, 2000). No caso apresentado, o resultado do histopatológico foi condizente com o diagnóstico de LPO, acompanhado dos seguintes achados: Hiperceratose, acantose, corpos de Civatte, como também a lâmina própria subjacente exibindo infiltrado inflamatório crônico contendo linfócitos, macrófagos e plasmócitos.

A etiopatogenia ainda é incerta, não existe um padrão a respeito dos aspectos histopatológicos da apresentação oral, e o prognóstico é considerado reservado, visto a inexistência de cura para a doença. As pesquisas apontam a imunidade mediada por células como mecanismo causador do dano aos ceratinócitos, com base na presença predominante de infiltrado linfocitário nos locais das lesões. A análise do sangue periférico de indivíduos com LPO proporcionou verificar diferenças na quantidade de células do sistema imunológico com relação aos níveis normais, correspondendo com a possibilidade de haver autoimunidade no LPO (Navas-Alfaro et al., 2003; Charazinska-Carewicz et al., 2008).

Razões psíquicas não só podem desencadear o processo de desenvolvimento do LPO para os predispostos às lesões, como acentuar, transformando lesões reticulares assintomáticas em erosivas e sintomáticas (Rodstrom et al. , 2001; Chiappelli, 1997). 
O líquen plano oral é uma patologia que apresenta um considerado desafio para sua identificação pelo profissional da saúde, como o cirurgião-dentista, em especial o estomatologista e o patologista. Essa peculiaridade se dá, principalmente, pela ausência de consenso clínico-histopatológico ao longo dos anos, fato observado em trabalhos previamente publicados, no qual muitas lesões sofriam transformação maligna, sem haver uma concordância com a lesão inicial, se líquen plano oral ou reação liquenoides (Van der Meij et al., 2003).

Mesmo aquelas lesões de líquen plano que não apresentem sintomatologia devem ser monitoradas, visto que existem indícios de que essas são lesões são passiveis de malignização (Huber, 2004). Partindo do princípio dessa malignização, sua causa ainda não está muito esclarecida na literatura, devido ao relato inadequado dos casos, entretanto estudos mostram uma percentagem de malignização que varia de $0 \%$ a $10 \%$, sendo a forma erosiva mais suscetível do que a forma reticular (Silverman et al., 1985; Hirota, 2007). As configurações incomuns atrófica, erosiva, em placa e as lesões localizadas na língua apresentaram considerado risco de transformação maligna. Corroborando a importância do diagnóstico preciso e do acompanhamento em longo prazo dos pacientes com LPO (Souza et al., 2005).

O tratamento do LPO não estabelece a cura da patologia, sendo agregado apenas à atenuação dos sinais e sintomas. É comparativamente satisfatório, porém de efeitos efêmeros. Lesões reticulares assintomáticas ordinariamente demandam apenas acompanhamento, enquanto o tratamento das formas atrófica e ulcerada se reserva ao alívio dos sintomas e eliminação das úlceras com propósito de reduzir o risco de malignização (Chan et al., 1999; Eisen et al., 2005).

Dessa maneira a abordagem terapêutica para a patologia tem por finalidade o alívio dos sintomas, partindo do pressuposto que tais lesões não admitem cura. Conseguinte a medicação de escolha baseia-se na administração de corticosteróide, o qual tem capacidade de modulara resposta inflamatória e imunológica. Entretanto, no presente caso não houve a administração medicamentosa, visto que as lesões regrediram no período de 2 meses, havendo a remissão dos sintomas nesse período devido a estabilização do estado psíquico e emocional do paciente.

Estratégias abordadas nas medidas intervencionais do paciente podem configurar uma melhora nos sinais e sintomas do paciente sem intervir diretamente na lesão, estratégias essas como, por exemplo, a adoção de exercício de relaxamento para aliviar a ansiedade tem permitido vivenciar um feedback bastante relevante, bem como a adesão de técnicas terapêuticas como ioga, meditação, acupuntura e shiatsu. As pessoas que adotam tais técnicas têm narrado uma sensação de maior bem-estar e, consequentemente, percebido melhoras significativas em sua saúde física (Silva, 2011).

$\mathrm{O}$ tratamento antidepressivo concerne-se em compreender de maneira integralizada, levando em consideração o ser humano em todas as suas prerrogativas, sejam estas incluídas nas esferas biológicas, psicológicas e sociais. Por conseguinte, a terapia deve abranger todas essas dimensões e lançar mão da utilização de estratégias conjuntas como psicoterapia, mudanças no estilo de vida e a terapia farmacológica (Thornhillet al., 2006).

Atualmente, tem-se vivenciado o avanço no tratamento farmacológico dos transtornos da ansiedade recomendado pelo médico, adotando alternativas como os benzodiazepínicos (BZD), todavia com a introdução da buspirona, esta é a primeira de uma classe de ansiolíticos, sendo as Azapironas o único fármaco dessa classe disponível no Brasil, o leque de medicamentos eficazes no transtorno de ansiedade vem sendo ampliado no percorrer dos anos (Andreatini et al., 2001).

\section{Conclusão}

Apesar da incerteza da etiopatogenia da LPO, fatores psicogênicos, como estresse e ansiedade, possuem grande influência na manifestação dessa lesão. Entretanto, mais estudos sobre essa temática devem ser realizados. 


\section{Referências}

Aguiar, C., Castro, T., Carvalho, A., Vale, O., Sousa, F., \& Vasconcelos, S. (2011). Antidepressantdrug. Acta Médica Portuguesa, 24 (1), 91-98. https://www.actamedicaportuguesa.com/revista/index.php/amp/article/view/339/109\%3e. http://dx.doi.org/10.20344/amp.339

Almeida, R. S., Guimarães, J. L., \& Almeida, J. Z. (2018). Estresse emocional e sua influência na saúde bucal. Revista DêCiênca em Foco, 2(1), 78-102. http://revistas.uninorteac.com.br/index.php/DeCienciaemFoco0/article/view/148

Andreatini, R., Boerngen-Lacerda, R., \& Zorzetto, F. D. (2001). Tratamento farmacológico do transtorno de ansiedade generalizada: perspectivas futuras. Revista Brasileira de Psiquiatria, $23 \quad$ (4), $\quad 233-242 . \quad$ https://www.scielo.br/scielo.php?pid=S151644462001000400011\&script=sci_abstract\&tlng=pt.10.1590/S1516-44462001000400011

Biaggio, A. M. B., Natalílio, L. (1979). Manual para o inventário de ansiedade traço-estado (IDATE). Centro de Psicologia Aplicada-CEPA

Boorghani, M., Gholizadeh, N., TaghaviZenouz, A., Vatankhah, M., \&Mehdipour, M. (2010). Oral lichen planus: clinical features, etiology, treatment and management; a review of literature. Journal of dental research, dental clinics, dental prospects, 4(1), 3-9. https://pubmed.ncbi.nlm.nih.gov/22991586/.10.5681 / joddd.2010.002

Canto, A.M., Müller, H., Freitas, R. R., \& Santos, P. S. S. (2010). Líquen plano oral (LPO): diagnóstico clínico e complementar. Anais Brasileiros de Dermatologia , 85(5), 669-675. https://www.scielo.br/scielo.php?script=sci_abstract\&pid=S0365-05962010000500010\&lng=en\&nrm=iso\&tlng=pt

Carrozzo, M., Thorpe, R. (2009). Oral lichen planus: a review. Minerva stomatologica, 58(10), 519-537. https://www.minervamedica.it/en/journals/minervastomatologica/article.php?cod=R18Y2009N10A0519

Cerqueira, J., Moura, J. R., Arsati, F., Lima-Arsati, Y., Bittencourt, R. A., \& Freitas, V. S. (2018). Psychological disorders and oral lichen planus: A systematic review. Journal of investigative and clinical dentistry, 9(4), 12363.https://pubmed.ncbi.nlm.nih.gov/30270524/. 10.1111/ jicd.12363

Chan, E. S. Y., Thornhill. M., \&Zakrzewska. J. J. M. (1999). Interventions for treating oral lichen planus.Cochrane Database of Systematic Reviews, 2. https://www.cochranelibrary.com/cdsr/doi/10.1002/14651858.CD001168/full 10.1002/14651858.CD001168.

Charazinska-Carewicz, K., Ganowicz, E., Krol, M., \& Gorska, R. (2008).Assessment of the peripheral immunocompetent cells in patients with reticular and atrophic-erosive lichen planus.Oral surgery, oral medicine, oral pathology, oral radiology, and endodontics, 105(2), 202-205. https://pubmed.ncbi.nlm.nih.gov/17656134/.10.1016/j.tripleo.2007.03.031

Chaudhary, S. (2004).Psychosocial stressors in oral lichen planus.Australian dental journal, 49(4), 192-195. https://pubmed.ncbi.nlm.nih.gov/15762340/. 10.1111/j.1834-7819.2004.tb00072.x

Chiappelli, F., Kung, M. A., Nguyen, P., Villanueva, P., Farhadian, E. A., \&Eversole, L. R. (1997). Cellular immune correlates of clinical severity in oral lichen planus: preliminary association with mood states. Oral diseases, 3(2), 64-70. https://pubmed.ncbi.nlm.nih.gov/9467344/.10.1111/j.1601-0825.1997.tb00014.x

Colonia, A., \& Vélez, L. F. (2011). Liquen Plano Oral. CES Odontología, 24 (2), 79-86. http://www.scielo.org.co/scielo.php?script=sci_arttext\&pid=S0120971 X2011000200009\&lng=en\&tlng=es

Danielli, J., Vilanova, L. S. R., Pedroso, L., \& Silva, M. A. G. (2010). Protocolo de Atendimento e Acompanhamento do Paciente com Líquen Plano Oral (LPO). Revista Odontologica do Brasil Central, 19(50), 233-238. https://www.robrac.org.br/seer/index.php/ROBRAC/article/view/471.

Dudhia, S. B., Dudhia, B. B., \& Shah, J. S. (2011).An Etiological, Clinical and Histological Study of Oral Lichen Planus with Comparative Evaluation between Various Therapies.Journal of Indian Academy of Oral Medicine and Radiology, 23, 507-512. https://www.researchgate.net/publication/269658473_An_Etiological_Clinical_and_Histological_Study_of_Oral_Lichen_Planus_with_Comparative_Evaluati on_between_Various_Therapies/citation/download.10.5005/jp-journals-10011-1211

Eisen, D., Carrozzo, M., Bagan S., J. V., \&Thongprasom, K. (2005), Número V Líquen plano oral: características clínicas e gestão. Oral Diseases, 11, 338349. https://onlinelibrary.wiley.com/action/showCitFormats?doi=10.1111\%2Fj.1601-0825.2005.01142.x. 10.1111/j.1601-0825.2005.01142.x

Eisenberg, E. (2000). Oral lichen planus: a benign lesion. Journal of oral and maxillofacial surgery: official journal of the American Association of Oral and Maxillofacial Surgeons, 58(11), 1278-1285. https://pubmed.ncbi.nlm.nih.gov/11078140/. 10.1053/joms.2000.16629

Fraga, H. F., Cerqueira, N. S., Ribeiro, L. S. F., Souza, S. E. Paraguassú, G. M., Filho, J. M. P., \& Sarmento, V. A. (2011). A importância do diagnóstico do líquen plano bucal. Journalofthe Health SciencesInstitute, 29, 27-30. https://www.unip.br/presencial/comunicacao/publicacoes/ics/edicoes/2011/01_janmar/V29_n1_2011_p27-30.pdf

Fitzpatrick, S. G., Hirsch, S. A., \& Gordon, S. C. (2014). The malignant transformation of oral lichen planus and oral lichenoid lesions: a systematic review. Journalofthe American Dental Association (1939), 145(1), 45-56. https://pubmed.ncbi.nlm.nih.gov/24379329/. 10.14219/jada.2013.10

Garrote, C. E. M., Teixeira, M. L., Ramacciato, J. C. (2010). Antidepressivos em odontologia: indicações e cuidados. Revista de Associação Paulista de Cirurgiões Dentistas, 64(4), 294-5. http://eduardojanuzzi.com.br/artigosRecomendados/2010/orientando_o_cd_2pag.pdf

Hampf, B. G., Malmström, M. J., Aalberg, V. A., Hannula, J. A., \&Vikkula, J. (1987). Psychiatric disturbance in patients with oral lichen planus. Oral surgery, oral medicine, and oral pathology, 63(4), 429-432. https://pubmed.ncbi.nlm.nih.gov/3472142/. 10.1016/0030-4220(87)90254-4

HIROTA, S. K. (2007). Líquen plano: etiopatogenia (Tese de Doutorado). Universidade de São Paulo-USP, São Paulo, SP, Brasil. https://www.teses.usp.br/teses/disponiveis/23/23139/tde-11042008-124535/publico/SilvioKenjiHirota.pdf

Huber M. A. (2004).Oral lichen planus.Quintessence international (Berlin, Germany: 1985), 35(9), 731-752. https://pubmed.ncbi.nlm.nih.gov/15470998/

Kurago, Z. B. (2016). Etiology and pathogenesis of oral lichen planus: an overview. Oral surgery, oral medicine, oral pathology and oral radiology, 122(1), 72-80. https://pubmed.ncbi.nlm.nih.gov/27260276/.10.1016/j.oooo.2016.03.011 
KIGNEL, S. (2007). Estomatologia: bases do diagnóstico para o clínico geral. Santos.

Lundqvist, E. N., Wahlin, Y. B., Bergdahl, M., \&Bergdahl, J. (2006). Psychological health in patients with genital and oral erosive lichen planus.Journal of the European Academy of Dermatology and Venereology: JEADV, 20(6), 661-666. https://pubmed.ncbi.nlm.nih.gov/16836492/. 10.1111/j.14683083.2006.01559.x

Medwar, C. V., \& Matheus, M. E. (2012). Antidepressivos Tricíclicos e Gabapentinóides: uma análise do perfil farmacológico no tratamento da dor neuropática. Revista Brasileira de Fármacia, 93, 290-297. https://www.scielo.br/scielo.php?script=sci_nlinks\&pid=S1981$8637201600040044700013 \& \operatorname{lng}=$ en

Mezzari, R., Iser, B. P. M. (2015). Desafios na prescrição de benzodiazepínicos em unidades básicas de saúde. Revista da Associação Médica do Rio Grande do Sul, 59(3), 198-203. http://www.amrigs.org.br/revista/59-03/07_1507_Revista\%20AMRIGS.pdf.

Navas-Alfaro, S. E., Fonseca, E. C., Guzmán-Silva, M. A., \&Rochael, M. C. (2003). Análise histopatológica comparativa entre líquen plano oral e cutâneo. Jornal Brasileiro de Patologia e Medicina Laboratorial, 39(4), 351-360. https://www.scielo.br/scielo.php?script=sci_arttext\&pid=S167624442003000400013. 10.1590/S1676-24442003000400013

Neville B. W., \&Damm, D. D. (2009). Patologia Oral e Maxilofacial (3a ed). Elsevier, 2009.

Pokupec, J. S., Gruden, V., \&Gruden, V., Jr (2009). Lichen ruberplanus as a psychiatric problem. PsychiatriaDanubina, 21(4), 514-516. https://pubmed.ncbi.nlm.nih.gov/19935485/

Radloff, LS (1977). A escala CES-D: uma escala de depressão de autoavaliação para pesquisa na população em geral. Applied Psychological Measurement, 1 (3), 385-401. https://journals.sagepub.com/doi/abs/10.1177/014662167700100306\#articleCitationDownloadContainer.10.1177/014662167700100306

Radwan-Oczko, M., Zwyrtek, E., Owczarek, J. E. \&Szcześniak, D. (2018).Perfil psicopatológico e qualidade de vida de pacientes com líquen plano oral. JournalofApplied Oral Science, 26, e20170, 146. ttp://www.scielo.br/scielo.php?script=sci_arttext\&pid=S1678-77572018000100411\&lng=en\&nrm=iso. $10.1590 / 1678-7757-2017-0$

Riga-Neto, A. A., Andrade, C. R., Navarro, C. M., Onofre, M. A., \&Massucato, E. M. S. (2013). Líquen plano e relação liquenóide: uma discussão diagnóstica. Revista de Odontologia da UNESP, 42, 1807-2577. https://www.revodontolunesp.com.br/article/5880197b7f8c9d0a098b518c.

Robledo-Sierra, J.,\& van der Waal, I. (2018). How general dentists could manage a patient with oral lichen planus. Medicina oral, patologia oral y cirugia bucal, 23(2), e198-e202. https://pubmed.ncbi.nlm.nih.gov/29476684/.

Rödström, P. O., Jontell, M., Hakeberg, M., Berggren, U., \&Lindstedt, G. (2001).Erosive oral lichen planus and salivary cortisol. Journal of oral pathology \& medicine: official publication of the International Association of Oral Pathologists and the American Academy of Oral Pathology, 30(5), 257-263. https://pubmed.ncbi.nlm.nih.gov/11334460/.10.1034/j.1600-0714.2001.300501.x

Schenkel, J. S., Lübbers, H. T., Rostetter, C., \& Metzler, P. (2017).Traitementmédicamenteux du lichen plan buccal.Swiss dental journal, 127(6), 538-539. https://pubmed.ncbi.nlm.nih.gov/28639683/.

Shirasuna, K. (2014). Oral lichen planus: Malignant potential and diagnosis. Journal Oral Science Internatinal, 11(1), 1-7. https://www.sciencedirect.com/science/article/pii/S134886431300030X. 10.1016/S1348-8643(13)00030-X

SILVA, A. B. B. (2011) Mentes ansiosas: medo e ansiedade além dos limites. Objetiva.

Silverman, S., Jr, Gorsky, M., \&Lozada-Nur, F. (1985). A prospective follow-up study of 570 patients with oral lichen planus: persistence, remission, and malignant association. Oral surgery, oral medicine, and oral pathology, 60(1), 30-34. https://pubmed.ncbi.nlm.nih.gov/3862010/. 10.1016/00304220(85)90210-5

Souza, F. A. C. G., \&Rosa, L. E. B. (2005). Perfil epidemiológico dos casos de líquen plano oral pertencentes aos arquivos da disciplina de patologia bucal da Faculdade de Odontologia de São José dos Campos - UNESP. Ciência Odontológica Brasileira, 8(4), 96-100. https://ojs.ict.unesp.br/index.php/cob/article/view/404. 10.14295/bds.2005.v8i4.404

Vallejo, M. J., Huerta, G., Cerero, R., \&Seoane, J. M. (2001). Anxiety and depression as risk factors for oral lichen planus.Dermatology (Basel, Switzerland), 203(4), 303-307. https://pubmed.ncbi.nlm.nih.gov/11752817/. 10.1159/000051777

Van der Meij, E. H., \& van der Waal, I. (2003).Lack of clinicopathologic correlation in the diagnosis of oral lichen planus based on the presently available diagnostic criteria and suggestions for modifications.Journal of oral pathology \&medicine : official publication of the International Association of Oral Pathologists and the American Academy of Oral Pathology, 32(9), 507-512. https://pubmed.ncbi.nlm.nih.gov/12969224/.10.1034/j.1600-0714.2003.00125.x

Venturini, D., Costa, J. R. S., Tibola, J., \&Tarquinio, S. B. C. (2006). Fatores Psicogênicos Associados ao Líquen Plano Bucal: Revisão da Literatura. Revista Odonto Ciência - Faculdade de Odontologia/Pontificia Universidade Católica do Rio Grande do Sul, $21(52)$, 191-198. https://pdfs.semanticscholar.org/d2ac/6183ccdf47f3c28247bd7d5e05b36e51a448.pdf

Vilanova, L. S. R., Danielli, J. Pedroso, L., \& Silva, M. A. G. S. (2012). Perfil epidemiológico de portadores de líquen plano oral atendidos no centro Goiano de doenças da boca (CGDB) - 12 anos de experiência. Revista Odontologica do Brasil Central, 21(59), 526-529. https://www.robrac.org.br/seer/index.php/ROBRAC/article/view/680/656

Volkweis, M. R., Blois, M. C., \& Zanin, R. F. (2015). Estudo retrospectivo de líquen plano bucal em um centro de especialidades odontológicas. Revista de Cirurgia e Traumatologia Buco-Maxilo-Facial, 15(2), 15-20. https://www.revistacirurgiabmf.com/2015/2/02-EstudoretrospectivodeLiquen.pdf

Tomaz, A. J., Willian, P. J., Quinto, J. H. S., Veltrini, V. C., Iwaki, L. C. V., \& Tolentino, E. S. (2015). Potencial de Transformación Maligna delLiquen Plano Oral: Estudio Retrospectivo. Internationaljournalofodontostomatology, 9(3), 511-517. https://scielo.conicyt.cl/scielo.php?script=sci_arttext\&pid=S0718381X2015000300025 10.4067/S0718-381X2015000300025 
Research, Society and Development, v. 10, n. 3, e43010313178, 2021

(CC BY 4.0) | ISSN 2525-3409 | DOI: http://dx.doi.org/10.33448/rsd-v10i3.13178

Thornhill, M. H., Sankar, V., Xu, X. J., Barrett, A. W., High, A. S., Odell, E. W., Speight, P. M., \& Farthing, P. M. (2006). The role of histopathological characteristics in distinguishing amalgam-associated oral lichenoid reactions and oral lichen planus.Journal of oral pathology \& medicine: official publication of the International Association of Oral Pathologists and the American Academy of Oral Pathology, 35(4), 233-240. https://pubmed.ncbi.nlm.nih.gov/16519771/. 10.1111/j.1600-0714.2006.00406.x

Warnakulasuriya, S. (2018).Clinical features and presentation of oral potentially malignant disorders.Oral surgery, oral medicine, oral pathology and oral radiology, 125(6), 582-590.R https://pubmed.ncbi.nlm.nih.gov/29673799/. 10.1016/j.oooo.2018.03.011

Werneck, J. T., Miranda, F. B., \& Silva, J. A. (2016). A. Desafios na distinção de lesões de Líquen Plano Oral e Reação Liquenóide. Revista Brasileira de Odontologia, 73(3), 247-52. http://www.revista.aborj.org.br/index.php/rbo/article/view/770. 10.18363/rbo.v73n3.p.247 